\title{
Astragaloside attenuates myocardial injury in a rat model of acute myocardial infarction by upregulating hypoxia inducible factor-1 $\alpha$ and Notch1/Jagged1 signaling
}

\author{
JUNMIN YU ${ }^{1}$, XIAOBO ZHANG $^{1}$ and YINA ZHANG ${ }^{2}$ \\ ${ }^{1}$ Department of Geriatrics, The Fourth Affiliated Hospital of Harbin Medical University, Harbin, Heilongjiang 150001; \\ ${ }^{2}$ Department of Geriatrics, The Second Affiliated Hospital of Harbin Medical University, \\ Harbin, Heilongjiang 150086, P.R. China
}

Received March 4, 2016; Accepted February 16, 2017

DOI: $10.3892 / \mathrm{mmr} .2017 .6522$

\begin{abstract}
The present study aimed to investigate the mechanisms underlying the cardioprotective effect of Astragaloside against myocardial injury following myocardial infarction (MI) in a rat model. Male Wistar rats were subjected to left anterior descending branch ligation. The rats that survived $24 \mathrm{~h}(\mathrm{n}=18)$ were randomly and equally assigned to three groups: MI model group, and 2.5 and $10 \mathrm{mg} / \mathrm{kg} / \mathrm{day}$ Astragaloside group. A further six rats underwent identical surgical procedures without artery ligation, serving as sham controls. Following 28 days of treatment, the left ventricle was harvested for morphological analysis, and mRNA and protein expression levels of hypoxia inducible factor-1 $\alpha$ (HIF-1 $\alpha$ ), Notch1 and Jagged1 were measured. Treatment with Astragaloside attenuated pathological changes in the myocardium. Compared with untreated MI rats, rats treated with Astragaloside exhibited significantly increased mRNA expression levels of HIF-1 $\alpha$, Notch1 and Jagged1 (all $\mathrm{P}<0.01$ ). HIF-1 $\alpha$ demonstrated a dose-dependent effect $(\mathrm{P}<0.05)$. Astragaloside $(10 \mathrm{mg} / \mathrm{kg} / \mathrm{day})$ significantly increased HIF-1 $\alpha$ $(\mathrm{P}<0.05)$, Notch1 $(\mathrm{P}<0.01)$ and Jagged1 $(\mathrm{P}<0.01)$ protein expression levels. Additionally, $2.5 \mathrm{mg} / \mathrm{kg}$ Astragaloside significantly increased Jagged1 protein expression levels compared with untreated MI rats. Furthermore, there was a dose-dependent effect of Astragaloside treatment $(\mathrm{P}<0.01)$. These findings suggested that the cardioprotective effects of Astragaloside against myocardial injury following MI may involve upregulation of HIF- $\alpha$, Notch1 and Jagged1 signaling, implicating these molecules as therapeutic targets for the treatment of MI.
\end{abstract}

Correspondence to: Dr Yina Zhang, Department of Geriatrics, The Second Affiliated Hospital of Harbin Medical University, 246 Xuefu Road, Harbin, Heilongjiang 150086, P.R. China

E-mail: yinazhangdc@sina.com

Key words: Astragaloside, myocardial injury, hypoxia inducible factor $1 \alpha$, Notch1, Jagged 1

\section{Introduction}

Acute myocardial infarction (MI) remains a primary cause of death among all types of cardiovascular disease. Early reperfusion via fibrinolysis or angioplasty is the predominant therapy to attenuate myocardial damage (1). Despite declining mortality rates in recent years, the prevalence of acute MI is increasing in the elderly population (2). Post-MI ischemia/reperfusion contributes to myocardial damage and pathological remodeling. Therefore, attenuating the extent of myocardial injury may be a useful strategy for the management of patients post-MI.

Myocardial ischemia/reperfusion may activate various molecular and cellular pathways that protect cardiomyocytes from ischemic injury (3). Among these signaling pathways, the Notch signaling pathway has been demonstrated to be involved in the process of cardiovascular disease. Notch proteins have been identified with at least five Notch ligands (Jagged1 and 2, and Delta1, 2 and 3) and 4 Notch receptors (Notch 1-4) (4). The Notch signaling pathway may be involved in infarct healing and cardiac repair $(5,6)$, and activation of Notch signaling may have a protective effect following cardiac injury (7-9). In addition, Notch signaling may activate the hypoxia-inducible factor- $1 \alpha$ (HIF-1 $\alpha$ ) gene via the Hes $1 /$ signal transducers and activators of the transcription 3 pathway (10). HIF-1 is a major regulator of the hypoxic response following MI (11) and serves a key role in regulating oxygen homeostasis. Activation of HIF-1 $\alpha$ by reduced oxygen or increased oxidative stress exerts a cardioprotective effect in the myocardium (12).

Astragaloside, the primary active constituent of Astragalus membranaceus (Huangqi), is widely used for the treatment of myocardial ischemic diseases in China (13-18). A previous study conducted by the authors (19) demonstrated that Astragaloside may attenuate post-MI myocardial ischemia in rats by promoting angiogenesis and upregulating vascular endothelial growth factor (VEGF) protein expression. However, the underlying mechanisms by which Astragaloside protects against myocardial injury in rats post-infarction remain to be fully elucidated. Little is understood about the effect of Astragaloside on HIF-1 $\alpha$ and the Notch1/Jagged1 signaling pathway. The purpose of the present study was to 
investigate the mechanisms underlying the cardioprotective effect of Astragaloside against myocardial injury in a post-infarction rat model, and the potential involvement of HIF-1 $\alpha$ and Notch1/Jagged1 signaling.

\section{Materials and methods}

Materials. Astragaloside was purchased from Chengdu Mansite Biological Technology Co., Ltd. (Chengdu, China). Polyclonal anti-HIF-1 $\alpha$ (cat. no. \#4426) and monoclonal anti-Notch1 (cat. no. \#3608) primary antibodies were purchased from Cell Signaling Technology, Inc. (Danvers, MA, USA), and monoclonal anti-Jagged1 (cat. no. ab124524) and anti-GAPDH (cat. no. ab181602) antibodies were purchased from Abcam (Cambridge, UK). A biotinylated horseradish peroxidase (HRP) -conjugated secondary antibody (cat. no. A0216) was purchased from Beyotime Institute of Biotechnology (Shanghai, China). TRIzol ${ }^{\circledR}$ reagent was obtained from Gibco; Thermo Fisher Scientific, Inc. (Waltham, MA, USA). Reverse transcription (RT), bicinchoninic acid protein (BCA) assay and $\mathrm{SYBR}^{\circledR}{ }_{\text {- }}$ Green Real-Time Polymerase Chain Reaction (PCR) kits were purchased from Thermo Fisher Scientific, Inc. Radioimmunoprecipitation assay (RIPA) lysis buffer was obtained from JRDUN Biotechnology (Shanghai, China).

Animals and experimental protocol. Male Wistar rats (mean weight, $250 \pm 30 \mathrm{~g}$; age, 2 to 3 months; $\mathrm{n}=45$ ) were supplied by the Changchun Yisi Experimental Animal Technology Co. (Changchun, China). They were housed at $22 \pm 2^{\circ} \mathrm{C}$ with 40-50\% humidity, a $12 \mathrm{~h} / 12 \mathrm{~h}$ light/dark cycle and free access to food and water. The study protocol was approved by the Ethical Committee of the Harbin Medical University (Harbin, China) and performed in accordance with the guidelines of Laboratory Animals published by the US National Institutes of Health.

Rats were randomly divided into sham control or acute MI groups. Animals were subjected to coronary artery ligation to induce acute MI as described previously (19). Briefly, rats were anesthetized with an intraperitoneal injection of $40 \mathrm{mg} / \mathrm{kg} 1 \%$ sodium pentobarbital (Merck KGaA, Darmstadt, Germany). Following tracheal intubation, all rats received positive pressure aerobic ventilation with a small animal ventilator (55-705B; Harvard University, Cambridge, MA, USA), and the chest was opened at the left fourth intercostal space. The heart was exteriorized following incision of the pericardium. A 6-0 silk suture was used to ligate the left anterior descending branch $\sim 3-5 \mathrm{~mm}$ distal to the aortic root. Successful generation of the acute MI model was verified by regional cyanosis of the infarct areas. Sham control animals were subjected to an identical surgical procedure without coronary artery ligation.

A total of 18 rats (35\% of rats died) surviving for $24 \mathrm{~h}$ were randomly assigned to three groups: MI model, $2.5 \mathrm{mg} / \mathrm{kg} / \mathrm{day}$ Astragaloside-treated, $10 \mathrm{mg} / \mathrm{kg} /$ day Astragaloside-treated or control ( $n=6 /$ group). Following surgery, all animals were kept in an air conditioned-controlled room at $22 \pm 2^{\circ} \mathrm{C}$ with $40-50 \%$ humidity in a $12 \mathrm{~h} \mathrm{light/dark} \mathrm{cycle} \mathrm{with} \mathrm{free} \mathrm{access}$ to water and standard rat feed. Astragaloside-treated rats were administered with the designated doses of Astragaloside by intraperitoneal injection for 28 days, whereas rats in the MI model and sham control groups received equal amounts of saline.

Gross cardiac morphology and histopathological examination. After 28 days of Astragaloside or saline treatment, rats were anesthetized with an intraperitoneal injection of $40 \mathrm{mg} / \mathrm{kg}$ $1 \%$ sodium pentobarbital. The heart was rapidly dissected and rinsed with saline, following which the left intraventricular pressure was maintained at $20 \mathrm{mmHg}$ by using water-filled balloon. The aortic arch, right ventricle, atrium and auricle were removed, and horizontally sectioned into five 1-mm thick sections. Infracted areas of the extracted left ventricle were fixed in $4 \%$ paraformaldehyde for $24 \mathrm{~h}$. The fixed tissue specimens were cut into 6- $\mu \mathrm{m}$ sections for hematoxylin-eosin (H\&E) staining. Morphological alterations of the stained tissues were assessed under a light microscope (CH20BIMF200; Olympus Corporation, Tokyo, Japan) at x400 magnification.

HIF-1 $\alpha$, Notch1 and Jagged1 mRNA expression. Reverse transcription-quantitative PCR (RT-qPCR) analysis was performed to measure HIF-1 $\alpha$, Notch1 and Jagged1 mRNA expression. Briefly, total RNA was extracted from $100 \mathrm{mg}$ samples of ischemic myocardium tissue with TRIzol reagent according to the manufacturer's protocol. The purity of RNA was measured according to the $\mathrm{A}_{260} / \mathrm{A}_{280}$ ratio spectrophotometrically. Total RNA ( $2 \mu \mathrm{g})$ from each sample was used to synthesize cDNA with Moloney Murine Leukemia Virus Reverse Transcriptase (Thermo Fisher Scientific, Inc.). The following primer sequences were synthesized by Shanghai Generay Biotech Co., Ltd. (Shanghai, China): Forward, 5'-CAG CGATGACACGGAAAC-3' and reverse, 5'-AGTGACTCT GGGCTTGAC-3' for HIF-1 $\alpha$; forward, 5'-CTTCGTGCTCCT GTTCTTTG-3' and reverse, 5'-GCCTCTGACACTTTGAAA CC-3' for Notch1; and forward, 5'-CACCCGAACTGGACA AAC-3' and reverse, 5'-AGCCTCAGACTGGGATAC-3' for Jagged1. These primer pairs resulted in 210, 105, and $170 \mathrm{bp}$ amplified products, respectively. Rat GAPDH was used as an internal control with the following primers (182 bp): Forward, 5'-GTCGGTGTGAACGGATTTG-3' and reverse, 5'-TCC CATTCTCAGCCTTGAC-3'. qPCR reactions were prepared using a SYBR Real-Time PCR kit. The PCR products were quantified by detecting the SYBR-Green fluorescence signal intensity and analyzed by Applied Biosystems Prism 7300 SDS software (Applied Biosystems; Thermo Fisher Scientific, Inc.). Relative mRNA expression levels of HIF-1 $\alpha$, Notch1 and Jagged 1 were quantified by the $2^{-\Delta \Delta \mathrm{Cq}}$ method, normalized to the levels of GAPDH.

HIF-1 $\alpha$, Notch1 and Jagged1 protein expression. HIF-1 $\alpha$, Notch1 and Jagged1 protein expression levels were measured by western blot analysis. Briefly, $20 \mathrm{mg}$ samples of frozen tissues were powdered and homogenized in 150-250 $\mu$ l RIPA lysis buffer. Protein levels were determined by performing a BCA assay according to the manufacturer's protocol. Prepared protein samples were separated by $10 \%$ SDS-PAGE and transferred onto nitrocellulose membranes. The membranes were blocked with milk powder in TBS for $60 \mathrm{~min}$ at room temperature, and subsequently incubated with anti-HIF-1 $\alpha$ (1:1,000 dilution), anti-Notch1 (1:1,000 dilution), anti-Jagged1 (1:500 dilution) and anti-GAPDH (1:1500 dilution) primary antibodies . 
Following overnight incubation at $4^{\circ} \mathrm{C}$, the membranes were washed three times with TBST (TBS with $0.1 \%$ Tween-20) and subsequently incubated with HRP-conjugated secondary antibodies (1:2,000 dilution) in TBS with $0.1 \%$ Tween-20 for $30 \mathrm{~min}$ at $37^{\circ} \mathrm{C}$. The bands were visualized using Enhanced Chemiluminescence reagents (EMD Millipore, Billerica, MA, USA), according to the manufacturer's protocol. The relative protein expression levels of HIF-1 $\alpha$, Notch1 and Jagged1 were normalized to the intensity of GAPDH bands from the same sample. All experiments were repeated in triplicate, and mean values were calculated.

Statistical analysis. Data are presented as mean \pm standard deviation. One-way analysis of variance followed by the Student-Newman-Keuls method was applied for multiple comparisons. All statistical analyses were performed using SPSS software version 17.0 (SPSS, Inc., Chicago, IL, USA). $\mathrm{P}<0.05$ was considered to indicate a statistically significant difference.

\section{Results}

Gross cardiac morphology. Heart structures were healthy in sham control rats (Fig. 1A), whereas untreated MI rats exhibited dilation of the heart, pale lesion sites, thinner ventricular walls and total ventricular wall involvement (Fig. 1B). In contrast, treatment with 2.5 (Fig. 1C) or $10 \mathrm{mg} / \mathrm{kg} /$ day (Fig. 1D) Astragaloside attenuated the dilation of heart and reduced the infarction size of MI rats.

Morphological analysis of myocardium treated with Astragaloside. Histopathological analysis of myocardial tissues was observed by H\&E staining. Myocardial tissues in control rats demonstrated apparent integrity of the myocardial cell membrane with no inflammatory cell infiltration (Fig. 2A). Myocardial tissues in infarct rats exhibited dissolved cardiomyocytes, distorted cardiac muscles, myocardial necrosis, a large number of fibroblasts or collagen fibers, and a small amount of inflammatory cell infiltration (Fig. 2B). In contrast, treatment with 2.5 (Fig. 2C) or $10 \mathrm{mg} / \mathrm{kg} / \mathrm{day}$ (Fig. 2D) Astragaloside attenuated the above histopathological abnormalities, and the boundary between the infarct area and non-infarct area was obvious.

Effects of Astragaloside on HIF-1 $\alpha$, Notch1, and Jaggedl $m R N A$ expression. As presented in Fig. 3A, no significant differences were observed in HIF-1 $\alpha$ mRNA expression levels in the myocardium of untreated MI rats compared with sham control rats $(1.7006 \pm 0.9756$ and $1.1495 \pm 0.6861$, respectively; $\mathrm{P}>0.05)$. Treatment with Astragaloside significantly increased HIF-1 $\alpha$ mRNA expression to $15.3790 \pm 4.2757$ at a dose of $2.5 \mathrm{mg} / \mathrm{kg} / \mathrm{day}$ and $20.8603 \pm 6.8249$ at $10 \mathrm{mg} / \mathrm{kg} /$ day (both $\mathrm{P}<0.01)$. Furthermore, HIF-1 $\alpha$ mRNA expression levels were significantly increased following treatment with $10 \mathrm{mg} / \mathrm{kg} /$ day Astragaloside compared with $2.5 \mathrm{mg} / \mathrm{kg} /$ day $(\mathrm{P}<0.05)$.

As presented in Fig. 3B, no significant differences were observed in Notch1 mRNA expression levels in the myocardium of untreated MI rats compared with sham control rats $(2.3227 \pm 0.6524$ and $1.8110 \pm 2.6816$, respectively; $\mathrm{P}>0.05)$. Treatment with Astragaloside significantly increased Notch1

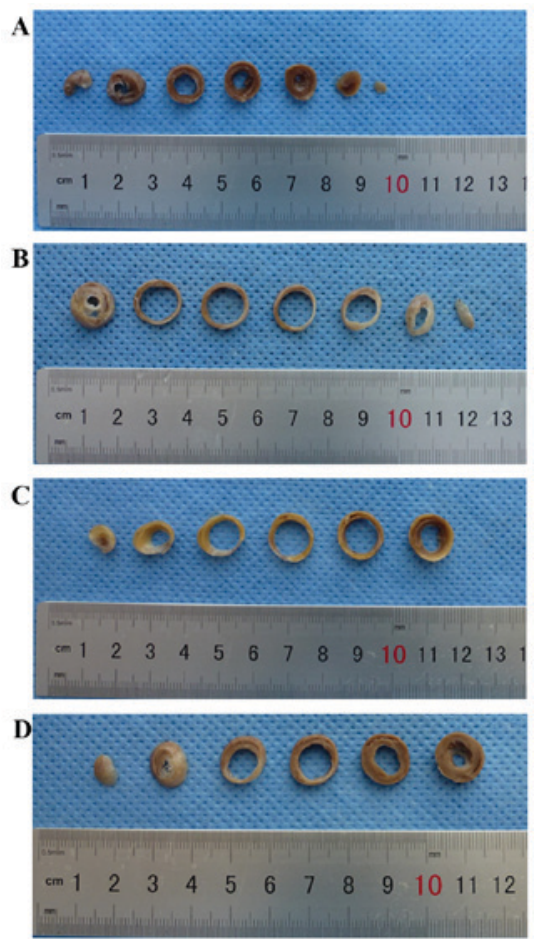

Figure 1. Gross cardiac morphology. (A) Rats in the sham control group exhibited a healthy heart structure. (B) Rats in the myocardial infarction model group exhibited increased dilation of the heart, pale lesion sites and thinner ventricular walls. Treatment with (C) 2.5 and (D) $10 \mathrm{mg} / \mathrm{kg} / \mathrm{day}$ Astragaloside significantly reduced the dilation of the heart and infarction size.

mRNA expression to $22.4513 \pm 5.0885$ at $2.5 \mathrm{mg} / \mathrm{kg} / \mathrm{day}$ and $26.3550 \pm 10.4480$ at $10 \mathrm{mg} / \mathrm{kg} /$ day compared with the expression level in MI model rats (both $\mathrm{P}<0.01$ ). However, no significant differences in Notch1 mRNA expression were observed between the different doses of Astragaloside $(\mathrm{P}>0.05)$.

As presented in Fig. 3C, no significant differences were observed in Jagged 1 mRNA levels in the myocardium of untreated MI rats compared with sham control rats $(1.5589 \pm 2.0397$ and $1.4880 \pm 1.5084$, respectively; $\mathrm{P}>0.05)$. Treatment with Astragaloside significantly increased Jagged1 mRNA expression to $8.4783 \pm 6.4066$ at $2.5 \mathrm{mg} / \mathrm{kg} /$ day and $12.1670 \pm 2.0818$ at $10 \mathrm{mg} / \mathrm{kg} /$ day (both $\mathrm{P}<0.01$ ). However, no significant differences were observed in Jagged1 mRNA expression between the different doses of Astragaloside $(\mathrm{P}>0.05)$.

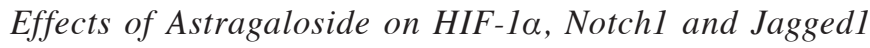
protein expression. Representative HIF-1 $\alpha$ protein bands with a molecular weight of $120 \mathrm{kDa}$ are presented in Fig. 4A. Myocardial HIF-1 $\alpha$ protein levels were significantly increased in MI model rats compared with sham control rats $(0.5668 \pm 0.1855$ and $0.3295 \pm 0.1553$, respectively; $\mathrm{P}<0.05)$. HIF-1 $\alpha$ protein expression levels following treatment with 2.5 and $10 \mathrm{mg} / \mathrm{kg} /$ day Astragaloside were 0.7259 \pm 0.2112 and $0.8009 \pm 0.2167$, respectively. Treatment with $10 \mathrm{mg} / \mathrm{kg} /$ day Astragaloside resulted in significantly increased HIF-1 $\alpha$ expression levels compared with MI model rats $(\mathrm{P}<0.05)$. HIF- $1 \alpha$ protein expression levels did not differ significantly between rats treated with different doses of Astragaloside ( $\mathrm{P}>0.05)$. 

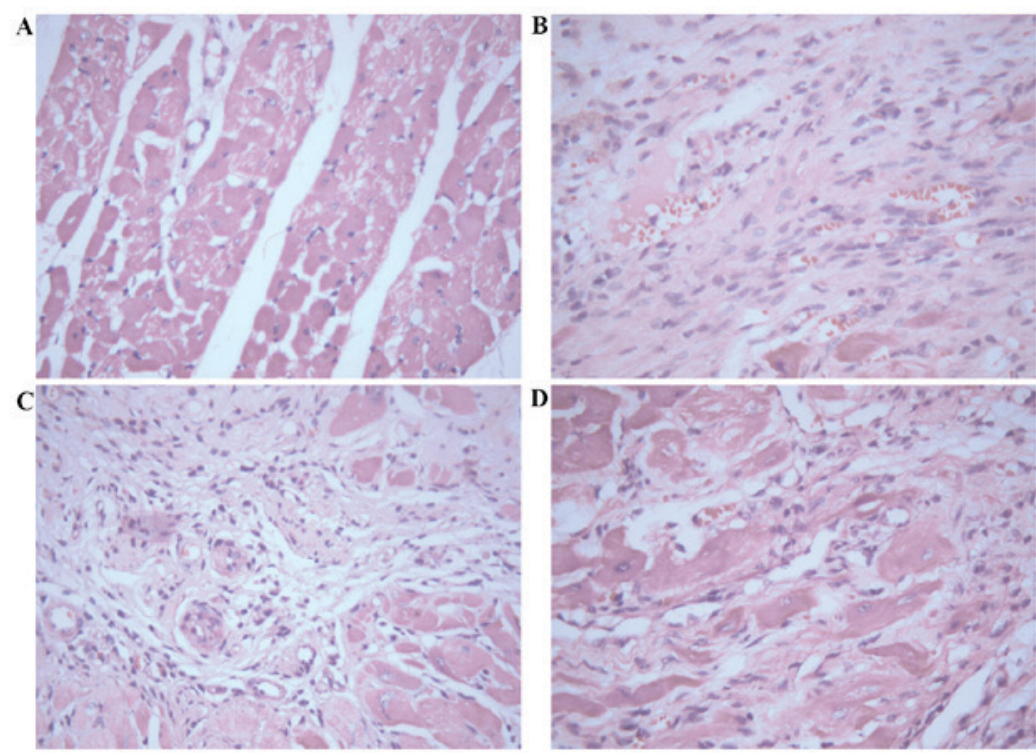

Figure 2. Morphological analysis of myocardial tissue stained with hematoxylin-eosin. (A) Rats in the sham control group exhibited apparent integrity of the myocardial cell membrane, with bright red cytoplasms and oval nuclei in the center of cells. (B) Rats in the myocardial infarction model group exhibited an irregular arrangement of myocardial cells, dissolved nuclei, a large number of fibroblasts or fibers, and ruptured of myocardial fibers. Treatment with (C) 2.5 and (D) $10 \mathrm{mg} / \mathrm{kg} / \mathrm{day}$ Astragaloside attenuated these histopathological alterations. Magnification, $\mathrm{x} 400$.
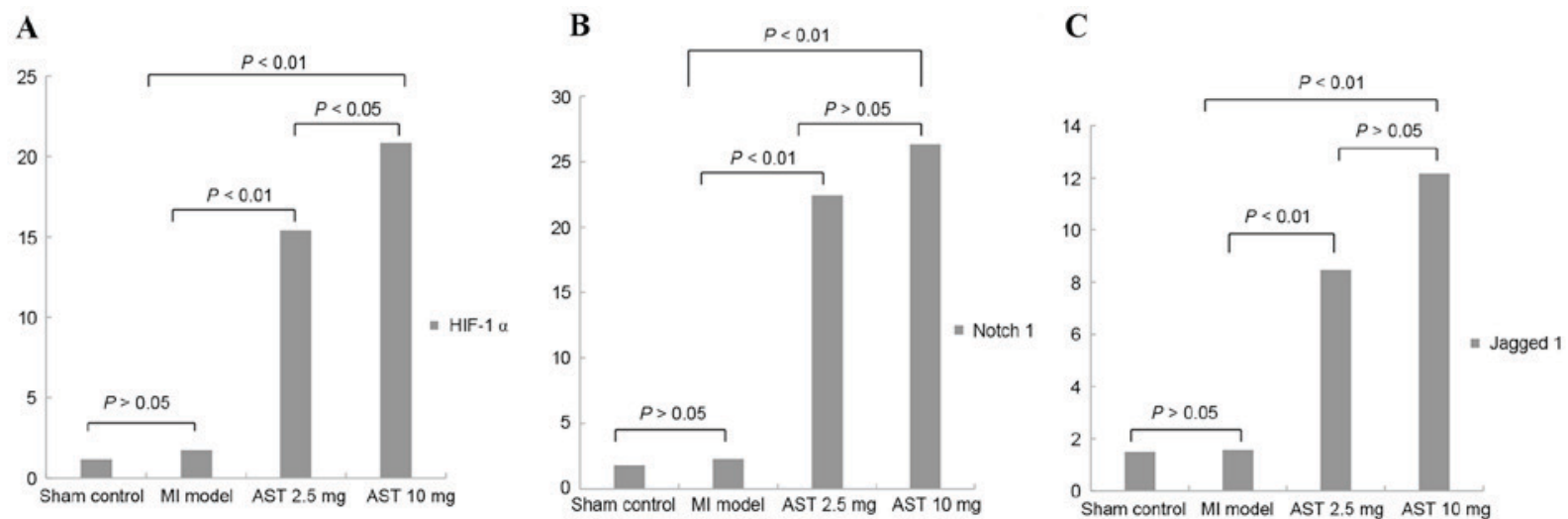

Figure 3. Effects of Astragaloside treatment on HIF-1 $\alpha$, Notch1 and Jagged1 mRNA expression levels. (A) HIF-1 $\alpha$, (B) Notch1 and (C) Jagged1 mRNA expression levels were determined by reverse transcription-quantitative polymerase chain reaction. Data are expressed as the mean \pm standard deviation ( $\mathrm{n}=6$ ). MI, myocardial infarction; AST, Astragaloside; HIF-1 $\alpha$, hypoxia-inducible factor 1- $\alpha$.

Representative Notch1 protein bands with a molecular weight of $120 \mathrm{kDa}$ are presented in Fig. 4B. Myocardial Notch1 protein expression levels were significantly increased in MI model rats compared with sham control rats $(0.7873 \pm 0.2207$ and $0.4781 \pm 0.1393$, respectively; $\mathrm{P}<0.05)$. Notch1 protein expression levels increased to $0.9567 \pm 0.2099$ and $1.1336 \pm 0.2014$ following treatment with 2.5 and with $10 \mathrm{mg} / \mathrm{kg} / \mathrm{day}$ Astragaloside, respectively. Treatment with $10 \mathrm{mg} / \mathrm{kg} / \mathrm{day}$ Astragaloside led to significantly upregulated Notch1 compared with the MI model rats $(\mathrm{P}<0.01)$. No significant differences in Notch1 protein expression levels were observed between the different doses of Astragaloside ( $\mathrm{P}>0.05)$.

Representative Jagged1 protein bands with a molecular weight of $134 \mathrm{kDa}$ are presented in Fig. 4C. Myocardial Jagged1 protein expression levels were significantly increased between MI model and sham control rats $(0.5244 \pm 0.0915$ and $0.3900 \pm 0.5689$, respectively; $\mathrm{P}<0.05)$. Treatment with 2.5 and $10 \mathrm{mg} / \mathrm{kg} /$ day Astragaloside significantly increased
Jagged 1 protein expression levels in rats $(0.7380 \pm 0.1091$ and $1.0580 \pm 0.1211$, respectively), compared with untreated MI rats $(\mathrm{P}<0.01)$. Furthermore, Jagged1 protein expression levels were significantly increased following treatment with $10 \mathrm{mg} / \mathrm{kg} / \mathrm{day}$ Astragaloside compared with $2.5 \mathrm{mg} / \mathrm{kg} / \mathrm{day}(\mathrm{P}<0.01)$.

\section{Discussion}

In the present study, a rat MI model was established via coronary artery ligation. Histological changes in the infarct hearts as observed by $\mathrm{H} \& \mathrm{E}$ staining presented dissolved or disrupted cardiomyocytes, a disorderly myocardial structure and a large number of fibroblasts or collagen fibers. Results of the gross study of the hearts revealed increased dilation of the heart, pale lesion sites, thinner ventricular walls or total ventricular wall involvement. Treatment with Astragaloside significantly attenuated these pathological abnormalities. In addition, Astragaloside treatment increased the mRNA and 
A

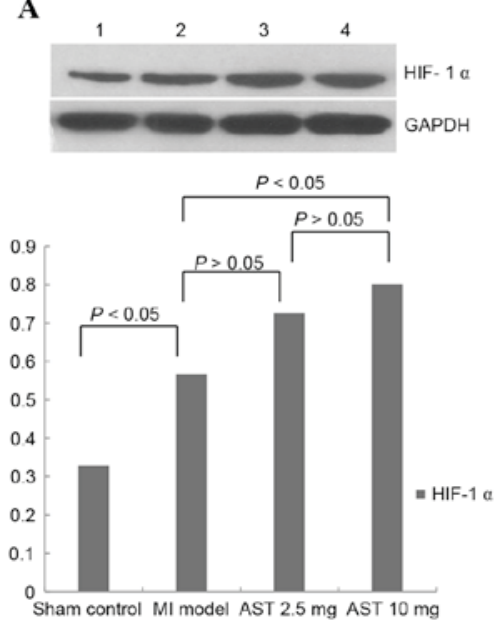

B

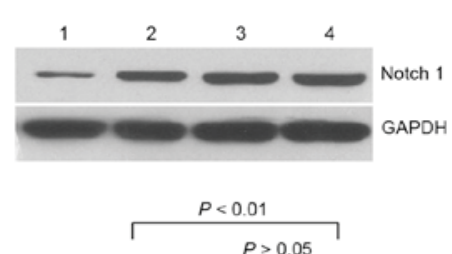

C

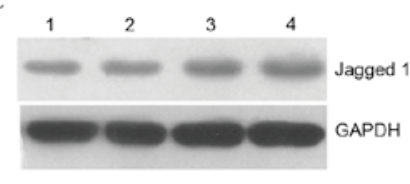

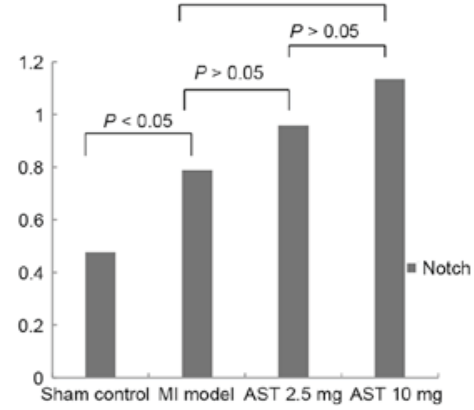

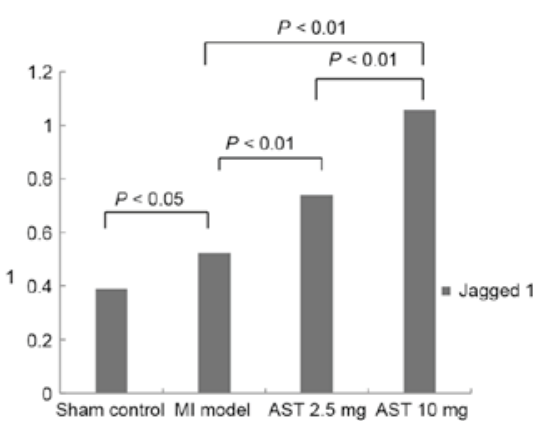

Figure 4. Effects of Astragaloside treatment on HIF-1 $\alpha$, Notch1 and Jagged1 protein expression levels. Representative western blotting images and quantification of (A) HIF-1 $\alpha$, (B) Notch1 and (C) Jagged1 protein expression levels. GAPDH served as an internal control. Data are expressed as the mean \pm standard deviation ( $\mathrm{n}=6$ ). 1, sham control group; 2, myocardial infarction model group; 3, $2.5 \mathrm{mg} / \mathrm{kg} / \mathrm{day}$ Astragaloside treatment group; 4 , $10 \mathrm{mg} / \mathrm{kg} / \mathrm{day}$ Astragaloside treatment group; MI, myocardial infarction; AST, Astragaloside; HIF-1 $\alpha$, hypoxia-inducible factor 1- $\alpha$.

protein expression levels of HIF-1 $\alpha$, Notch1 and Jagged1 to certain degrees; however, these results were dose-dependent. These findings indicated that the cardioprotective effect of Astragaloside might be partially mediated by HIF-1 $\alpha$ and the Notch1/Jagged1 signaling pathway.

HIF, a nuclear transcriptional regulatory factor, consists of HIF- $1 \alpha$ and HIF-1 $\beta$. HIF-1 $\alpha$ is sensitive to hypoxia and stabilizes under such conditions. It is widely understood that hypoxia serves a key role in the process of myocardial ischemia. Higher HIF-1 $\alpha$ expression is observed in the early (20) and late (21) stages of myocardial ischemia. MI induces upregulation of HIF-1 $\alpha$ in ischemic or hypoxic myocardium, which contributes to cardioprotective effects $(20,22,23)$. Persisting ischemia at the site of infarction may persist for numerous days to a later phase. Constitutive overexpression of HIF-1 $\alpha$ attenuates the infarct size and improves cardiac function 4 weeks post-infarction (24). Thus, HIF-1 $\alpha$ has been considered as a therapeutic target for cardioprotection (25). In the present study, treatment with 2.5 and $10 \mathrm{mg} / \mathrm{kg} /$ day Astragaloside significantly increased HIF-1 $\alpha$ mRNA expression levels compared with untreated MI rats $(\mathrm{P}<0.01)$. HIF-1 $\alpha$ protein expression levels were significantly increased in the $10 \mathrm{mg} / \mathrm{kg} /$ day Astragaloside group than the untreated $\mathrm{MI}$ rats $(\mathrm{P}<0.05)$. However, obvious dose-dependent effects of Astragaloside treatment were observed at the mRNA level $(\mathrm{P}<0.05)$.

The Notch signaling pathway is crucial for the regulation of cellular proliferation, differentiation and apoptosis. A hypoxic environment in the myocardium may lead to Notch signaling activation, which attenuates myocardial injury and improves cardiac function by promoting myocardial regeneration, protecting ischemic myocardium or inducing angiogenesis (26). Notch1 and Jagged1 are the predominant forms of Notch signaling expressed in adult myocytes (27). Furthermore, Notch 1 signaling is activated in proliferating embryonic and immature cardiomyocytes (8) or in the border zone of the myocardium following infarction (7). Notch1 signaling additionally inhibits cardiomyocyte apoptosis in ischemic postconditioning (28). Consistently, myocardial injury may be attenuated by activation of Notch1 via exogenous administration of Jagged1 (29). In the current study, Notch1 and Jagged1 expression in the myocardium was detected 4 weeks after MI. Administration of 2.5 or $10 \mathrm{mg} / \mathrm{kg} /$ day Astragaloside significantly increased Notch1 and Jagged1 mRNA expression levels compared with MI model rats (both $\mathrm{P}<0.01$ ). Compared with $\mathrm{MI}$ model rats, treatment with $10 \mathrm{mg} / \mathrm{kg} /$ day Astragaloside significantly increased Notch1 protein expression $(\mathrm{P}<0.01)$. However, significant differences in Jagged1 protein expression levels were observed following administration with Astragaloside at either dose (both $\mathrm{P}<0.01$ ). Furthermore, there were statistically significant differences in Jagged1 protein expression levels between the different doses of Astragaloside $(\mathrm{P}<0.01)$. Taken together, the above findings suggested that upregulation of Notch1/Jagged 1 signaling by Astragaloside may be the underlying mechanism whereby Astragaloside attenuates myocardial injury following MI.

Numerous studies have investigated the cardioprotective effects of Astragaloside. A previous study of the authors demonstrated that Astragaloside improves myocardial ischemia post-MI by inducing angiogenesis in the ischemic myocardium of rats (19). Astragaloside additionally may improve ischemic scope, epicardial ECG and myocardial enzymes in the acute MI dog heart (13). The cardioprotective effects of Astragaloside may be linked to the inhibition of calcium overload in cardiac myocytes (30), decrease in myocardial apoptosis and transforming grow th factor- $\beta$ expression (17), increase in protein expression levels of VEGF (19), or to the antioxidant properties of Astragaloside (18). Overall, these data supported the potential cardioprotective effects of Astragaloside.

There are numerous limitations to the present study. Infarction repair is a dynamic process, but the time-dependent changes in HIF-1 $\alpha$, Notch1, and Jagged1 expression were not investigated. Secondly, although the present study demonstrated the cardioprotective effects of Astragaloside administration in the ischemic myocardium, the dose-dependent effect was 
not obvious. The dose-dependent effects of Astragaloside on HIF-1 $\alpha$ and Jagged1 protein and mRNA expression levels were not consistent in this study. These inconsistent findings may be associated with degradation of the extracted protein samples or other upstream controlling genes that are involved in the regulation of mRNA and protein expression. The mechanisms underlying the regulation of HIF-1 $\alpha$, Notch1 and Jagged1 expression require further investigation.

In conclusion, the present study demonstrated that administration of Astragaloside for 28 days was associated with an improvement in myocardial injury in rats post-infarction. In addition, Astragaloside treatment increased myocardial expression levels of HIF-1 $\alpha$, Notch1 and Jagged1. These findings indicated that the cardioprotective effects of Astragaloside may be mediated partially via the upregulation of HIF- $\alpha$ and Notch1/Jagged1 signaling, implicating them as therapeutic targets for the treatment of MI.

\section{Acknowledgements}

The present study was supported by the Heilongjiang Provincial Department of Education (grant no. 12521297).

\section{References}

1. Schwartz Longacre L, Kloner RA, Arai AE, Baines CP, Bolli R, Braunwald E, Downey J, Gibbons RJ, Gottlieb RA, Heusch G, et al: New horizons in cardioprotection: Recommendations from the 2010 National Heart, Lung and Blood Institute Workshop. Circulation 124: 1172-1179, 2011.

2. Forouzanfar MH, Moran AE, Flaxman AD, Roth G, Mensah GA, Ezzati M, Naghavi M and Murray CJ: Assessing the global burden of ischemic heart disease, part 2: Analytic methods and estimates of the global epidemiology of ischemic heart disease in 2010. Glob Heart 7: 331-342, 2012.

3. Zamilpa R and Lindsey ML: Extracellular matrix turnover and signaling during cardiac remodeling following MI: Causes and consequences. J Mol Cell Cardiol 48: 558-563, 2010.

4. Ishitani T, Hirao T, Suzuki M, Isoda M, Ishitani S, Harigaya K, Kitagawa M, Matsumoto K and Itoh M: Nemo-like kinase suppresses Notch signalling by interfering with formation of the Notch active transcriptional complex. Nat Cell Biol 12: 278-285, 2010.

5. Gude N and Sussman M: Notch signaling and cardiac repair. J Mol Cell Cardiol 52: 1226-1232, 2012.

6. Li Y, Hiroi Y and Liao JK: Notch signaling as an important mediator of cardiac repair and regeneration after myocardial infarction. Trends Cardiovasc Med 20: 228-231, 2010.

7. Gude NA, Emmanuel G, Wu W, Cottage CT, Fischer K Quijada P, Muraski JA, Alvarez R, Rubio M, Schaefer E and Sussman MA: Activation of Notch-mediated protective signaling in the myocardium. Circ Res 102: 1025-1035, 2008.

8. Kratsios P, Catela C, Salimova E, Huth M, Berno V, Rosenthal N and Mourkioti F: Distinct roles for cell-autonomous Notch signaling in cardiomyocytes of the embryonic and adult heart. Circ Res 106: 559-572, 2010.

9. Li Y, Hiroi Y, Ngoy S, Okamoto R, Noma K, Wang CY, Wang HW, Zhou Q, Radtke F, Liao R and Liao JK: Notch1 in bone marrow-derived cells mediates cardiac repair after myocardial infarction. Circulation 123: 866-876, 2011.

10. Lee JH, Suk J, Park J, Kim SB, Kwak SS, Kim JW, Lee CH, Byun B, Ahn JK and Joe CO: Notch signal activates hypoxia pathway through HES1-dependent SRC/signal transducers and activators of transcription 3 pathway. Mol Cancer Res 7: 1663-1671, 2009.

11. Lee SH, Wolf PL, Escudero R, Deutsch R, Jamieson SW and Thistlethwaite PA: Early expression of angiogenesis factors in acute myocardial ischemia and infarction. N Engl J Med 342: 626-633, 2000

12. Li X, Zhao H, Wu Y, Zhang S, Zhao X, Zhang Y, Wang J, Wang $\mathrm{J}$ and Liu $\mathrm{H}$ : Up-regulation of hypoxia-inducible factor-1 $\alpha$ enhanced the cardioprotective effects of ischemic postconditioning in hyperlipidemic rats. Acta Biochim Biophys Sin (Shanghai) 46: 112-118, 2014.
13. Chunli L, Yu C, Wenwei L, Haitao Y, Biao S and Shijie Y: Effects of Astragalus saponins on ischemic scope, epicardial ECG, myocardial enzymes in acute myocardial infarcted dog heart. Baiqiuen Yike Daxue Xuebao 21: 111-113, 1995 (In Chinese)

14. Zhang Z, Chen LX, Qin LM and B J: Effect of Astragalus on hemodynamics and oxygen free radical in myocardial ischemia reperfusion injury in rats. Chin J Info Trad Chin Med 7: 7-8, 2000 (In Chinese).

15. Feng L, Meng D, Chen XJ, Yang D and Zhang JN: Protective action of Astragalosides on myocardial injury induced by isoproterenol in rats. Chin Pharm J: 1313-1316, 2006 (In Chinese).

16. Wang $\mathrm{N}$, Zhu $\mathrm{K}$, Yue $\mathrm{H}$, et al: Protective effects of Astragalus saponins on cardiomyocytes injury induced by oxidative stress. J Hebei Trad Chin Med Pharmacol 22: 8-9, 2007 (In Chinese).

17. Jia H, Wweiping J, Jiajie L and WZ Li: Protective effect of astragalosides on myocardial damage in diabetic mice. Acta Universitatis Medicinalis Anhui 290-294, 2012.

18. Han D, Zhang YW, Liu M, et al: The hemodynamic and antioxidant effects of Astragaloside on ventricular remodeling rats. Chin J Lab Diag: 17:1956-1959, 2013. (In Chinese)

19. Cao BD, Jiang W, Wang HD, Wang LY, Yu JM, Zhang XB: The effect of Astragalus on VEGF in ischemic myocardium of rats. Chin J Crit Care Med: 1033-1035, 2014.

20. Blanco Pampín J, García Rivero SA, Otero Cepeda XL, Vázquez Boquete A, Forteza Vila J and Hinojal Fonseca R: Immunohistochemical expression of HIF-1alpha in response to early myocardial ischemia. J Forensic Sci 51: 120-124, 2006.

21. Parisi Q, Biondi-Zoccai GG, Abbate A, Santini D, Vasaturo F, Scarpa S, Bussani R, Leone AM, Petrolini A, Silvestri F, et al: Hypoxia inducible factor-1 expression mediates myocardial response to ischemia late after acute myocardial infarction. Int J Cardiol 99: 337-339, 2005.

22. Adluri RS, Thirunavukkarasu M, Dunna NR, Zhan L, Oriowo B, Takeda K, Sanchez JA, Otani H, Maulik G, Fong GH and Maulik N: Disruption of hypoxia-inducible transcription factor-prolyl hydroxylase domain-1 (PHD-1-/-) attenuates ex vivo myocardial ischemia/reperfusion injury through hypoxia-inducible factor- $1 \alpha$ transcription factor and its target genes in mice. Antioxid Redox Signal 15: 1789-1797, 2011.

23. Poynter JA, Manukyan MC, Wang Y, Brewster BD, Herrmann JL, Weil BR, Abarbanell AM and Meldrum DR: Systemic pretreatment with dimethyloxalylglycine increases myocardial HIF-1alpha and VEGF production and improves functional recovery after acute ischemia/reperfusion. Surgery 150: 278-283, 2011.

24. Kido M, Du L, Sullivan CC, Li X, Deutsch R, Jamieson SW and Thistlethwaite PA: Hypoxia-inducible factor 1-alpha reduces infarction and attenuates progression of cardiac dysfunction after myocardial infarction in the mouse. J Am Coll Cardiol 46: 2116-2124, 2005

25. Ong SG and Hausenloy DJ: Hypoxia-inducible factor as a therapeutic target for cardioprotection. Pharmacol Ther 136: 69-81, 2012.

26. Zhou XL and Liu JC: Role of Notch signaling in the mammalian heart. Braz J Med Biol Res 47: 1-10, 2014.

27. Croquelois A, Domenighetti AA, Nemir M, Lepore M, Rosenblatt-Velin N, Radtke F and Pedrazzini T: Control of the adaptive response of the heart to stress via the Notch1 receptor pathway. J Exp Med 205: 3173-3185, 2008.

28. Yu B and Song B: Notch 1 signalling inhibits cardiomyocyte apoptosis in ischaemic postconditioning. Heart Lung Circ 23: 152-158, 2014.

29. Pei H, Yu Q, Xue Q, Guo Y, Sun L, Hong Z, Han H, Gao E, $\mathrm{Qu}$ Y and Tao L: Notch1 cardioprotection in myocardial ischemia/reperfusion involves reduction of oxidative/nitrative stress. Basic Res Cardiol 108: 373, 2013.

30. Meng D, Chen XJ, Yang D, Bian YY, Li P and Zhang JN: Effects of Astragaloside on the cultivation of calcium overload in rat cardiac myocytes. Chin J Clin Rehab 8: 462-463, 2004. 